\title{
ABOUT DARSY'S LAW DURING FLUIDS MOTION IN THE MICRO-CRACKED CHANNELS
}

\author{
Maleyka Mammadova \\ Department of Engineer and Computer Graphics \\ Azerbaijan State Oil and Industry University \\ 20 Azadlig ave., Baku, Azerbaijan, AZ 1010 \\ mamedova-1944@mail.ru
}

\begin{abstract}
Firstly it has been experimentally revealed that during fluid motion in the micro-cracked channel and in the equivalent porous medium an unknown additional resistance arises in the scientific technical literature that is the "microcrack-fluid" effect. It has been demonstrated that the determined "microcrack-fluid" effect is the cause of linear Darcy's law violation in the micro-cracked channels.

It has been revealed in the work that during fluids moving in the microcracked channel there is a critical size of crack for the homogeneous fluid (water, viscous and anomalous fluids) and a hydrodynamic effect as so-called "microcrack-fluid" is manifested.

So for the first time we determined the critical value of opening - her on the basis of experimental investigations in cracks. It was found that at $h<h_{c r}$ the anomalous properties are manifested for viscous fluids and rheological parameters are increased for anomalous fluids, and at $h \geq h_{c r}$ these effects disappear. It has been established that the reason of the anomalous behavior of fluids in the microcrack with $h<h_{c r}$ opening is the effect occurred in the "microcrack-fluid" system.

It is shown that microcrack with certain opening can be considered as a model but the ultra-low permeable porous medium is nature.

It has been determined that the critical value of the Reynolds number calculated for viscous and abnormal fluids in the microcracked channel and in the equivalent porous medium in the microcrack is $\operatorname{Re}<1$.

The new fact about Darcy's law violation during fluids flow in microcrack with $\mathrm{h}<\mathrm{hcr}$ opening has been experimentally revealed i.e. micro-cracked effect of "microcrack-fluid" system is a cause of Darcy's law violation.

It is recommended to taking into consideration the microcracked effect in the "fluid-medium" system for regulation and creation of the new technical and technological processes in the different branches of industry.

Keywords: micro-crack channel, microcrack opening, Newtonian and non-Newtonian fluids, limiting yield stress, structural viscosity, "microcrack-fluid" effect.
\end{abstract}

DOI: $10.21303 / 2461-4262.2020 .001386$

\section{Introduction}

As it is known there is a law proving that the volume of water flowing through a sand layer with known properties is proportional to pressure and inversely proportional to the sand layer thickness [1-9].

The actual geometry of the microcrack space can be equivalent to the corresponding porous medium therefore it is advisable to conduct the experiments in microcracks and to use these results in the corresponding porous medium.

As it is known with increasing filtration rate the Darcy's law is violated. The experiments descriptions according to determination of the critical Reynolds number, wherein Darcy's law is violated, have been published in many articles, but there isn't indication of the Darcy's law violation in the porous medium and in the equivalent microcrack channels in these works. For example the results of experiments [2] with sand and gravel mixture have shown that three ranges can be distinguished:

- corresponding to Darcy's law being laminar for $\operatorname{Re}<5$;

- transitional at $\mathrm{Re}=5-2 \cdot 10^{2}$;

- turbulent $\operatorname{Re}>2 \cdot 10^{2}$.

In this work it has been shown as well that ground water filtration is considered as the increased instability movement. The disturbances leading to origin of the local turbulence at low filtration rates can occur at the grains edges during obligatory grinding of the cross section. There is the deviation from the linear Darcy's law at $\mathrm{Re}>5$ in the authors' experiments. 
The review of works [3] shows that the values of the critical Reynolds number obtained by various researchers differ in hundreds of times.

The experimental data indicating of Darcy's law violation in the field of small pressure gradients are convincing $[4,5]$.

The numerous problems having focused theoretical and practical values have been solved in the field of fluid and gas mechanics and the various hydrodynamic effects have been discovered which have both scientific and national economic importance. The new effects will be discovered in this area of science and practice.

In the technological literature, much valuable material has been accumulated on the movement of fluids in channels of various cross-sections. However, the analysis of numerous materials, namely monographs, textbooks, articles and other works, showed that among these works there are no studies devoted to the motion of various liquids in channels with a micron cross section. There are numerous theoretical and experimental works in the literature confirming that with a decrease in the size of the channel cross-section, a quantitative inconsistency between the calculations of theoretical and experimental data was found, and this is explained by the fact that in experiments on the channel walls, various layers are formed, which reduce the cross-sectional area of the channels [1-9].

In these studies, there is no consensus on the manifestation of anomalous behavior of fluids in microcracks [1-9]. None of these works indicate the influence of the size of the crack opening on the features of the fluid flow, that is, these properties are taken regardless of the opening. For this reason, this effect has not been fully taken into account in industrial processes and in the practice of extracting oil from such reservoirs.

The aim of research is to create a consensus on the manifestation of anomalous behavior of fluids in channels with micron openings, to study the effect of "microcrack-liquid" in microcracks and low-permeability porous media, and to try to use the effect in various fields of engineering and technology.

Darcy's law violation can be arisen mostly due to the "microcrack-fluid" effect.

The results of experimental studies of the viscous and anomalous fluids motion in plane-parallel and plane-radial cracks with micron opening are presented below for confirming the Darcy's law violation according to the opening value of microcracked channels [10, 11].

\section{Materials and methods of investigation of the linear Darcy's law violation}

The structure of plane-parallel and plane-radial cracked channels and the experimental technique are given in works $[10,11]$.

The cracks of the given thickness are created by layerings located between the plates. In such a case the error of opening value is $0.1 \mu \mathrm{m}$. The pressure distribution in the crack was controlled by holes located along the radius. During the experimental investigation the pressure gauges reading installed in all holes were controlled that confirmed the absence of flow discontinuity.

The experimental installation with the elements was created in a way that it would be able to provide the flow continuity in the slot and to control the presence or the absence of flow continuity in the slot during experiment.

With the use of standard pressure gauges with $\delta=(0.2 \div 0.35) \%$ error the different pressure drops were created in the microcrack. The appropriate volumetric fluid flow rates $Q$ were measured according to reaching steady-state mode.

The measurements were made by purpose-made U-tube pressure gauges as a check on. It was established that the measurements were within accuracy used by pressure gauges.

The mass flow rate was determined on electronic scale with an accuracy up to $0.1 \mathrm{mg}$. The relative error for the velocity gradient and limiting yield stress in cracks are $\gamma=(2 \div 3) \%$ and $(0.2 \div 1.2) \%$, respectively.

The experiments were begun with slow opening, with $10 \mu \mathrm{m}$, and in some cases with $5 \mu \mathrm{m}$, and the rheological dependencies for viscous fluid were nonlinear. Then with the increasing of opening to some value of opening and even larger crack sizes, the rheological dependencies became linear, we named this opening value as critical opening for this fluid. 
To eliminate secondary factors, the installation was developed with consideration to the following requirements:

- providing the leak-proofness of installation;

- observance of constancy at all points of crack opening, pressure and temperature in the installation;

- observance of providing the flow continuity in the slot;

- control of the presence or absence of different layers on the crack walls surfaces;

- control of cleaning and control of the fractured volume of the test liquid.

The fractured reservoir is represented by two plates. The inner surface of plates was processed and grinded with accuracy corresponding to 10 category, with thickness of $4 \cdot 10^{-2} \mathrm{~m}$, which were made of stainless steel. The layings between the plates were both leak-proofing material and for formation of crack with the suitable size [10].

With the objective of providing crack undeformability the plates were made of $40 \mathrm{X}$ steel which had surface hardness of 40-50 units according to Rockwell after heat treatment of HFC (high frequency current). The indicating gauge fixed on the upper plate of model was used to control the crack deformation.

The experiments were carried out in microcracks with opening of $10 \div 240 \mu \mathrm{m}$ with water, kerosene, viscous and anomalous oil.

The experimental investigations were carried out under steady-state conditions in isothermal conditions, the temperature permanence was maintained by the ultra thermostat.

The crack saturation by the studied fluid was carried out under slight pressure with simultaneous vacuum evaporation.

For revealing of investigated fluids in plane and plane radial cracks the results were processed in the coordinates of $\gamma-\tau$, where the average shear rate is $\gamma=6 \mathrm{Q} / \mathrm{Fh}$ in the plane cracks and $\gamma=Q / 4 \pi r h^{2}$ in the radial; shear stress is $\tau_{w}=\Delta P h / 2 L$ in the plane cracks and $\tau_{w}=\Delta P h / L$ in the radial.

Newtonian oil in the microcrack $\left(h<h_{c r}\right)$ are described by either power law model or by Shvedov-Bingham model and the flow in microcrack $\left(h \geq h_{c r}\right)$ is described by the parameters of fluids in bulk.

Taking into consideration the fact that the engineering oilfield problems usually occurs at high velocity gradients therefore Shvedov-Bingham model has been used in work for experimental results processing i.e. the mechanical property of the fluid in the cracks is characterized by limiting shear stress $\tau_{0 h}$ and apparent viscosity $\mu$. The limit shear stress $\tau_{\mathrm{oh}}$ and apparent viscosities $\mu_{h}$ at different temperatures and the crack opening have been determined on the basis of the $\gamma=\gamma(\tau)$ curves for Newtonian and non-Newtonian fluids.

\section{Research results}

\section{1. Fluids motion in the microcracked channels}

On the base of experimental investigations in microcracked channels there have been developed new approaches to the fluid mechanics in cracks of channels and their application in different fields of technology, as well as for effectiveness increase of oil field development in fractured and ultra-low permeable porous media $[10,11]$.

During the motion of water, viscous and anomalous oil in micron size cracks and equivalent ultra-low permeable porous medium, the water and viscous oil take nature of the anomalous fluid and anomalous fluids even more increase the rheological parameters. Such physical effect of "microcrack-fluid" causes an additional resistance in the ultra-low permeable porous medium.

Therefore, it is necessary to develop a method to reduction the channels size from $h \leq h_{c r}$ condition to $h>h_{c r}$ condition for the decrease of the additional resistance of fluids flow in the microcracks, or to reduce the rheological parameters of fluid and both at the same time that can be achieved by use of strong physical, wave and thermal fields.

The $\gamma$ dependence on $\tau$ during water motion in plane-parallel and plane-radial microcracks in the different values of the crack opening quantity at $303 \mathrm{~K}$ temperature is presented in Fig. 1, 2. 
As can be seen from these figures the non-Newtonian properties, that decrease with the crack opening increase, are manifested during water movement both in rectangular and plane-radial cracks $\left(h<h_{c r}\right)$. The limiting shear stress equals to zero and the viscosity remains constant at the opening values of 22 and $25 \mu \mathrm{m}$ and higher at 313 and $306 \mathrm{~K}$ temperatures, respectively. During water movement in plane-radial microcrack at opening values of 30 and $35 \mu \mathrm{m}$ at 303 and $293 \mathrm{~K}$ temperature the limiting shear stress equals to zero and the apparent viscosity remains constant both in the first and in the second series of experiments.

The obtained dependencies $\gamma=f(\tau)$ of Newtonian oil in plane-parallel microcracks at different values of crack opening at $303 \mathrm{~K}$ temperature are shown in Fig. 3. As can be seen in Fig. 3 with increasing crack opening the shear stress is decreased during Newtonian oil movement but at $130 \mu \mathrm{m}$ opening the shear stress equals to zero and the viscosity remains constant at 306 temperature.

One can be seen in Fig. 4, 5 that in the case of non-Newtonian oil flow in plane-parallel and plane-radial cracks with opening increasing the limiting yield stress and apparent viscosity of oil are decreased up to certain crack value. The limiting series the limiting yield stress and apparent viscosity do not depend on $h$ and remain constant at $303 \mathrm{~K}$ temperature at $180 \mu \mathrm{m}$ opening values. shear stress equals to zero and the apparent viscosity remains constant during anomalous oil flow in plane-parallel crack at 130 and $160 \mu \mathrm{m}$ opening values at 313 and $306 \mathrm{~K}$ temperatures, respectively [11]. So it has been experimentally determined that there is a critical value $\left(h_{c r}\right)$ of opening quantity below which the limiting yield stress $\tau_{\mathrm{o} h}$ and apparent viscosity $\mu_{h}$ are appreciably increased. It has also been established that during the Newtonian fluids motion in cracks at $h<h_{c r}$ there are manifested the non-Newtonian properties and with increase of the crack opening $h$ up to $h_{k p}$ non-Newtonian properties are decreased but and at $h<h_{c r}$ these properties are disappeared. But during the non-Newtonian fluids motion the anomalous properties are increased and with increasing crack opening they are decreased up to the initial value.

The data of the non-linearity of rheological dependence for viscous and anomalous fluid are of special interest.

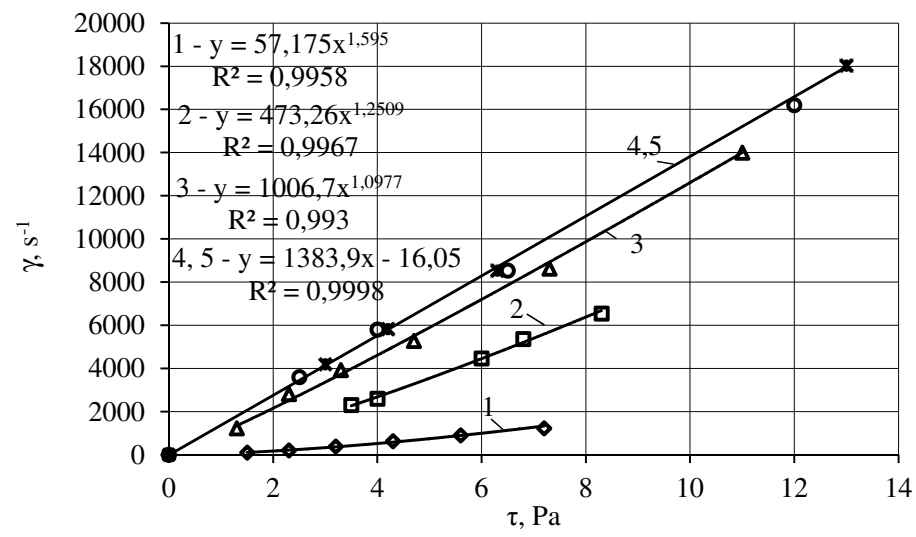

Fig. 1. The dependence of $\gamma$ from $\tau$ during water movement in plane-parallel cracks $(T=303 \mathrm{~K})$, at opening values, microns: 10 - curve $1 ; 15$ - curve $2 ; 20$ - curve $3 ; 25,30$ - curve 4,$5 ; h_{c r}=25 \mu \mathrm{m}$

As seen from Fig. 1-5 at $h \geq h_{c r}$ for different opening-crack values, all points of the $\gamma=f(\tau)$ dependences for viscous (straight line 4,5) and anomalous fluid (curve 4-6) respectively are presented on the same line both viscous and anomalous fluid. It proves the reliability of the specified critical values of the crack opening.

So for the first time we determined the critical value of opening $-h_{c r}$ on the basis of experimental investigations in cracks. It was found that at $h<h_{c r}$ the anomalous properties are manifested for viscous fluids and rheological parameters are increased for anomalous fluids, and at $h \geq h_{c r}$ these effects disappear. It has been established that the reason of the anomalous 
behavior of fluids in the microcrack with $h<h_{c r}$ opening is the effect occurred in the "microcrack-fluid" system.

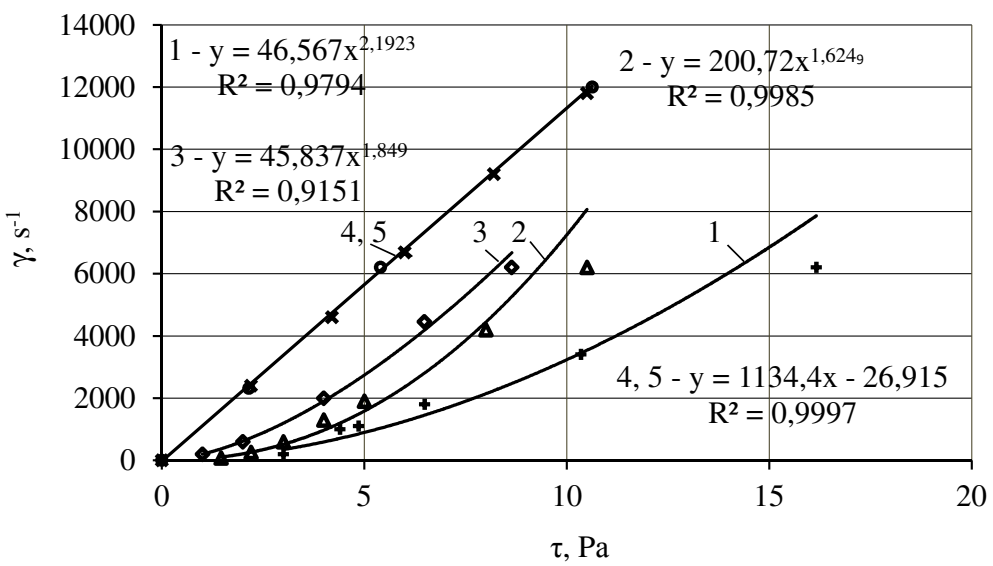

Fig. 2. The dependence of $\gamma$ from $\tau$ during water movement in plane-radial cracks ( $T=303 \mathrm{~K}$ ), at opening values, microns: 10 - curve $1 ; 15$ - curve $2 ; 20$ - curve $3 ; 30,35$ - curve 4,$5 ; h_{c r}=30 \mu \mathrm{m}$

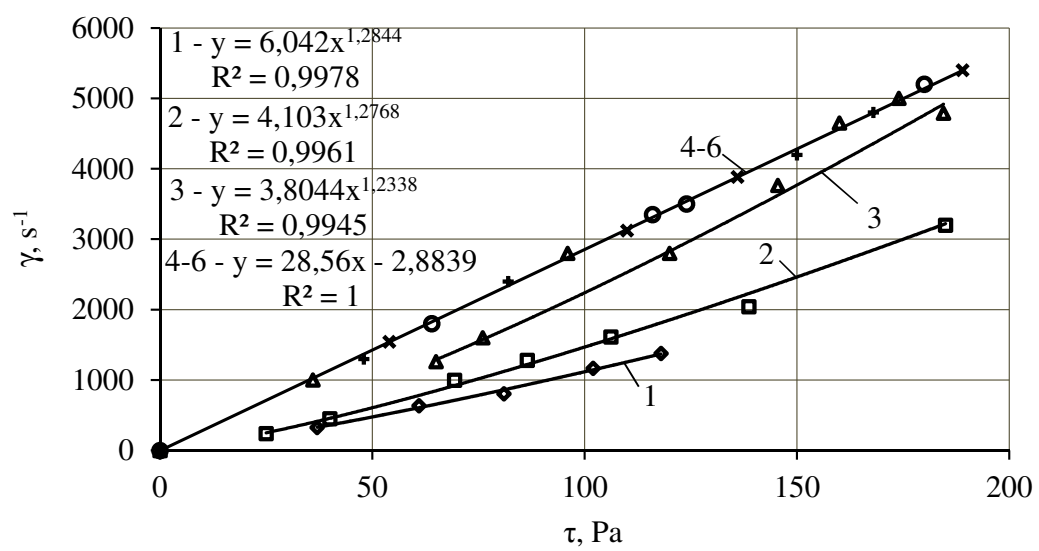

Fig. 3. The dependence of $\gamma$ from $\tau$ during Newtonian movement in plane-parallel cracks ( $T=303 \mathrm{~K}$ ), at opening values, microns: 40 - curve 1; 65 - curve 2; 90 - curve 3; $130,140,160$ - straight line $4-6 ; h_{c r}=130 \mu \mathrm{m}$

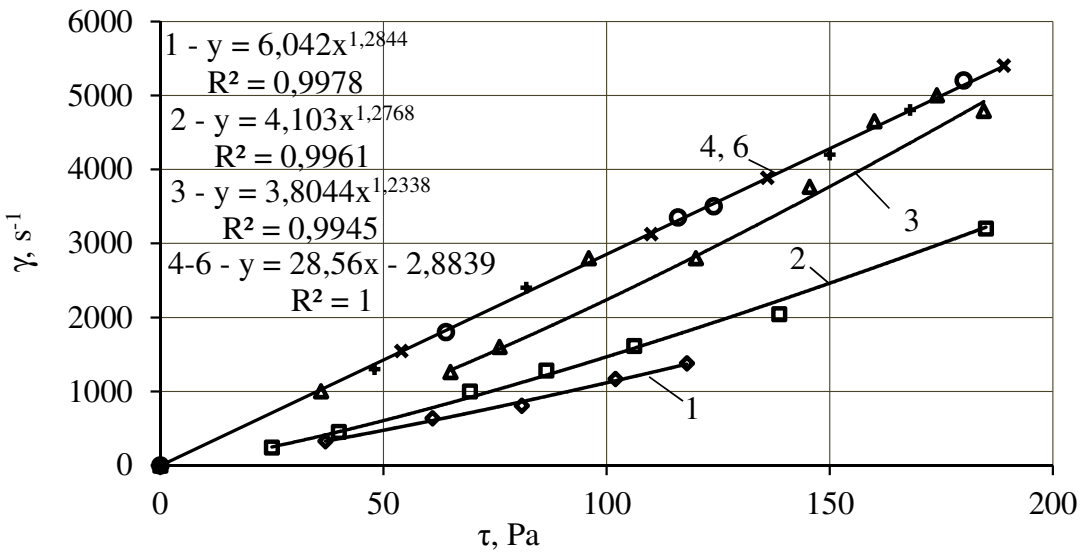

Fig. 4. The dependence of $\gamma$ from $\tau$ during anomalous oil to plane-parallel microcracks ( $T=303 \mathrm{~K}$ ) at different values of the crack opening, microns: 65 - curve $1 ; 90$ - curve $2 ; 130$ - curve 3; $160,170,180$ - curve $4-6 ; h_{c r}=160 \mu \mathrm{m}$ 


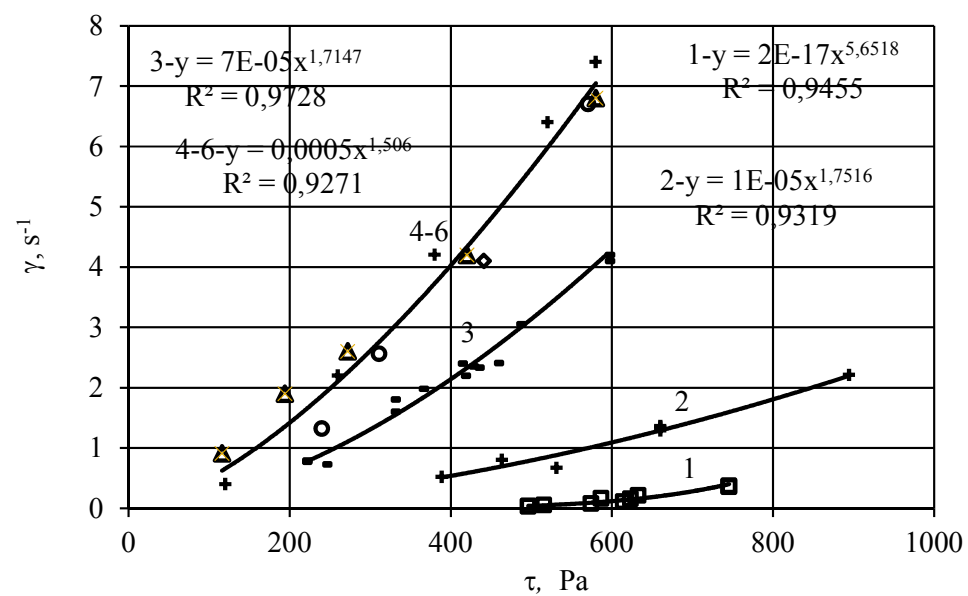

Fig. 5. The dependence of $\gamma$ from $\tau$ anomalous during oil in plane-radial cracks $(\mathrm{T}=303 \mathrm{~K})$ at different values f crack opening, microns: 90 - curve $1 ; 120$ - curve $2 ; 160$ - curve 3); $180,220,240-$ curve $4-6 ; h_{c r}=180 \mu \mathrm{m}$

\section{2. The estimation of the Reynolds number}

For determining the Reynolds parameters according to Genki-Ilyushin formula the calculations were made on the base of the experimental data of the flow of viscous fluid and anomalous oil in the plane-radial cracks.

The following below-mentioned equations formulas were used for viscous (at $h<h_{c r}$ ) and viscous-plastic fluids (at $h<h_{c r}$ and $h>h_{c r}$ ) and for viscous (at $h>h_{c r}$ ) fluid:

$$
\begin{gathered}
\mathrm{Re}=\frac{v \rho l / \mu}{\left(1+\frac{\tau_{0 h}}{\mu v}\right)}, \\
\operatorname{Re}=\frac{v \rho l}{\mu},
\end{gathered}
$$

where $v$ is speed; $l$ is the lateral length of the plane-radial crack; $\rho$ is the water density at $t=303 \mathrm{~K}$; $\mu$ is water viscosity at $h<h_{c r} ; \tau_{0 h}$ is limiting yield stress at $h<h_{c r}$.

The estimations of the experimental results show that the critical value of the Reynolds number calculated by formula (1) for viscous (at $h<h_{c r}$ ) and anomalous (at $h \leq h_{c r}$ and $h>h_{c r}$ ) fluids in the microcrack is $\operatorname{Re}<1$. And the values of the Reynolds number for viscous fluid calculated by the formula (2) for crack opening $h \geq h_{c r}$ are within [12] work for slots with smooth walls.

The microcrack with defined opening can be considered as a model of ultra-low permeable porous medium.

The calculations of the coefficient determination of loss under friction along the crack length were made by Darcy-Weisbach formula.

The dependencies of $\lambda$ Re from Re constructed on log scale during the viscous fluid flow in the plane-radial microcrack with the opening of $10 \mu \mathrm{m}$ in different pressure drops values are showed in Fig. 6. As it is shown from Figure the increasing of the Reynolds number allows to increasing of the coefficient of loss under friction along the crack length.

The following dependence was obtained to establish the transition dependencies from model to nature with the permanent viscous and anomalous fluids flow.

$$
\sqrt{k}=a h-b h_{c r}
$$

The values of $a$ and $b$ coefficients during motion of the viscous and anomalous fluids in plane-radial cracks are presented in the Table 1. 


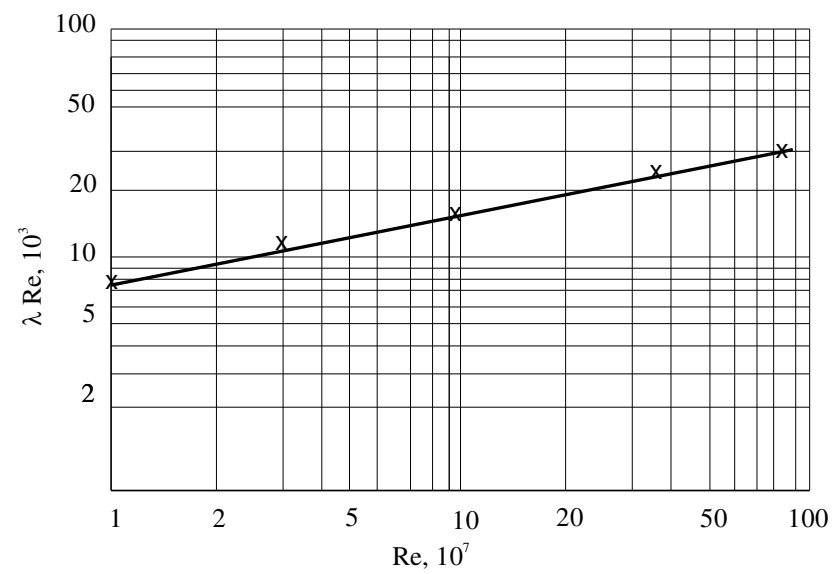

Fig. 6. The dependence of $\lambda \operatorname{Re}$ from Re during the viscous fluid flow in plane-radial microcracks with the crack opening of $10 \mu \mathrm{m}$ at various pressure drops values

Table 1

The values of $a$ and $b$ coefficients in plane-radial cracks

\begin{tabular}{ccccc}
\hline & \multicolumn{3}{c}{ Coefficients } \\
\hline \multirow{2}{*}{ Fluid } & \multicolumn{2}{c}{ At injection } & \multicolumn{2}{c}{ At production } \\
\cline { 2 - 5 } & $\mathbf{a}$ & $\mathbf{b}$ & $\mathbf{a}$ & 0.0180 \\
\hline For viscous fluid & 0.0709 & 0.0181 & 0.0667 & 0.0638
\end{tabular}

\section{Results discussion}

The manifestation of the "microcrack-liquid" effect could be explained as an analogy of the Euler stability of the rod.

From the models of the anomalous behavior of fluids it follows that in crack with small thickness the fluid under the action of the forces applied at the ends of crack in the conditions of comparatively low average shear rates can behaves like a stable compressed rod and has certain form of equilibrium in crack. The stable rod-shaped form of fluid equilibrium depends on the crack thickness. If the thickness of thin crack is small then applied forces at the crack ends press the fluid rod to the crack walls. The changes occur in the fluid jet properties due to changing its structure and friction forces on the walls. With increasing crack thickness the micro-cracked effect disappears. Between these two equilibrium states there exists so-called critical wherein fluid can preserve initial properties. This crack thickness is called as the critical.

Change in the mechanical properties of fluids when moving in channels, the dimensions of which are $h \leq h_{c r}$ and their recovery when moving in channels, the dimensions of which $h>h_{c r}$ represent the effect of "microcrack-fluid" $h>h_{c r}$. It does this by using strong physical fields. Consequently, for the activation of moving and resting fluids in microcracks, the dimensions of the channels are necessary from the state $h \leq h_{c r}$ to the state $h>h_{c r}$.

In this regard, we have a number of specific checks:

- measures to increase the success rate using hydraulic fracturing and hydro-acid fracturing;

- impact of vibration waves to enhance the flow of fluid to the bottom of the wells;

- impact on the bottomhole zone of wells.

This means that it is advisable to use strong physical fields to increase the oil recovery factor.

Thus, the found "microcrack-fluid" effect is a cause of violation of the linear Darcy law in microcrack channels. The value of the Reynolds number is established for a viscous and anomalous fluid in microcrack channels and an equivalent porous medium of less than unity.

The developed technique allows appreciating the critical value of the crack opening in the CCD and the relevant permeability of the porous medium according to the data of the steady-state 
condition of well survey. The CCD permeability assessment is of great importance for efficiency increasing of different stimulation methods on CCDs.

The obtained results will be used in the different branches of industry including the following:

- in the field of science "Mechanics of fluid, gas and plasma" - it is necessary to introduce a new section "Mechanics of fluid and their mixtures in a microcrack";

- oil industry - it is possible to achieve the maximum increase of oil recovery due to the involvement of the oil from the ultra-low permeable beds' zones in the development;

- machinery manufacturing and technology - developing a new approach to the design of lubricating systems of machines and mechanisms and to the selection of oils;

- medicine - developing a new approach to the processes of formation and cleaning of blood clots in the human vessels.

The results of the conducted investigations require taking into consideration the fractured effect at the parameters' assessment of the technological processes' system and technical devices of "microcrack-fluid" that has scientific and practical bearing on various branches of industry, machinery manufacturing, instrumentation, chemical technologies and medicine.

\section{Conclusions}

For the first time, it was experimentally revealed that the cause of the manifestation of anomalous properties of viscous fluids, in particular water, and the enhancement of these properties for anomalous fluids in cracks, is the new microcrack effect of the "microcrack-fluid" system we discovered.

It is shown that microcrack with certain opening can be considered as a model but the ultralow permeable porous medium is nature.

The new fact about Darcy's law violation during fluids flow in microcrack with $h<h_{c r}$ opening has been experimentally revealed i.e. micro-cracked effect of "microcrack-fluid" system is a cause of Darcy's law violation.

A criterion for the applicability of Darcy's law was established for studying the processes of motion of viscous and anomalous fluids in a channel with microcracks and in an equivalent porous medium at values of the Reynolds parameter more than unity.

It has been determined experimentally that non-Newtonian properties are manifested during water and viscous fluids movement in microcrack with $h<h_{c r}$ opening, i.e. one-parameter fluid behaves like two-parameter anomalous fluid in microcrack with $h<h_{c r}$ opening and fluid recovers its one-parameter properties at $h \geq h_{c r}$. Abnormal two-parameter fluid in microcrack with opening $h<h_{c r}$ behaves like two-parameter abnormal fluid with increased rheological parameters and it restores its rheological properties in microcrack with $h \geq h_{c r}$ opening.

The assessment of the critical value of the crack opening corresponding to the equivalent effective permeability of the porous medium allows to determine the presence of the "microcrack-fluid" effect at the investigated objects of oil fields.

A new effect "microcrack-liquid" was discovered, which makes additional resistance to the flow of liquids in microchannels.

Taking into account various technological processes of industry, especially in the oil industry, since the use of the effect of "microcrack-liquid" in the oil industry will put an end to the concept of "unrecoverable reserves" of oil.

\section{References}

[1] Mirzadzhanzade, A. H., Barenblatt, G. I., Entov, V. M., Zheltov, Yu. V., Margulov, G. D., Rassohin, G. V., Ryzhik, V. M. (1970). $\mathrm{O}$ vozmozhnom vliyanii nachal'nogo gradienta na razrabotku mnogoplastovyh gazovyh i gazokondensatnyh mestorozhdeniy pri vodonapornom rezhime. Izvestiya vysshih uchebnyh zavedeniya. Neft' i gaz, 1, 39-45.

[2] Mirzadzhanzade, A. H., Mingareev, R. Sh., Entov, V. M., Grayfer, V. I., Vahitov, G. G., Diyashev, R. N., Zaytsev, Yu. V. (1972O nelineynoy fil'tratsii v sloistyh plastah, 1, 44-49. Available at: https:/oil-industry.net/Journal/archive_detail.php?ID= 5738\&art $=105169$

[3] Scheidegger, A. E. (1954). Statistical Hydrodynamics in Porous Media. Journal of Applied Physics, 25 (8), $994-1001$. doi: https://doi.org/10.1063/1.1721815 
[4] Kotov, A. I., Nerpin, S. V. (1958). Vodoupornye svoystva glinistyh pochv i gruntov i priroda nachal'nyh gradientov fil'tratsii. Izvestiya AN SSSR, OTN, 9, 106-119.

[5] Deryagin, B. V., Churaev, N. V. (1987). Structure of water in thin layers. Langmuir, 3 (5), 607-612. doi: https://doi.org/10.1021/ la00077a002

[6] Korotenko, V. A., Grachev, S. I., Kushakova, N. P., Sabitov, R. R. (2014). Physical models of viscous-plastic oils displacement. Neftepromyslovoe delo, 5, 5-10. Available at: https://www.elibrary.ru/item.asp?id=21478987/

[7] Grachev, S. I., Korotenko, V. A., Kushakova, N. P., Zotova, O. P. (2016). The question of the displacement of oil from the anomalous collectors. Advances in current natural sciences, 10, 114-118.

[8] Zakirov, S. N., Barenbaum, A. A., Zakirov, E. S., Indrupskiy, I. M., Serebryakov, V. A., Klimov, D. S. (2016). Revisiting the Development of Oil Deposits with Low Permeability Reservoirs. Indian Journal of Science and Technology, 9 (42). doi: https:// doi.org/10.17485/ijst/2016/v9i42/104219

[9] Liu, W., Yao, J., Chen, Z., Liu, Y. (2015). Effect of quadratic pressure gradient term on a one-dimensional moving boundary problem based on modified Darcy's law. Acta Mechanica Sinica, 32 (1), 38-53. doi: https://doi.org/10.1007/s10409-0150526-2

[10] Mamedova, M. A., Gurbanov, R. S. (2015). Investigation of the Rheology of Fluids in Fracture and Pore Channels and Determination of Their Opening. Journal of Engineering Physics and Thermophysics, 88 (4), 815-824. doi: https://doi.org/10.1007/ s10891-015-1256-9

[11] Mammadova, M., Gurbanov, R. S. (2017). Hydromechanical substantiation of the microcrack-fluid effect. Mechanics, 22 (6). doi: https://doi.org/10.5755/j01.mech.22.6.12649

[12] Pavlov, I. A. (2008). Experimental investigations of the nonlinear filtration in the faulted mountain massif. Journal "Mining informational analytical bulletin", 2, 302-308.

Received date 20.03.2020

(C) The Author(s) 2020

Accepted date 26.08.2020

This is an open access article under the CC BY license

Published date 30.09.2020

(http://creativecommons.org/licenses/by/4.0). 